\title{
Visualization of femtosecond laser-induced stress anisotropy in amorphous and crystalline materials
}

\author{
Ben McMillen ${ }^{1, a}$, Feng Chen ${ }^{2}$ and Yves Bellouard ${ }^{1}$ \\ ${ }^{1}$ Galatea Lab, STI/IMT École Polytechnique Fédérale de Lausanne, Rue de Maladière 71b, CH-2002 Nuechâtel, Switzerland \\ ${ }^{2}$ Shandong University, School of Physics, C Block, Zhixin Building, 27 Shanda Nanlu, Jinan, Shandong, 250100, China
}

\begin{abstract}
In recent years, micro manufacturing with femtosecond lasers has received considerable attention as an efficient technique for producing three-dimensional devices, combining multiple functionalities in a single monolithic substrate. In this manufacturing process, stress-anisotropy resulting from non-ablative laser exposure can have both positive and negative effects on the process out-come. In this work, we present a simple method for visualizing stress anisotropy, combining highly symmetric laser-written patterns with polarization microscopy, as a tool for identifying the various anisotropic contributions to the laser fabrication process.
\end{abstract}

\section{Introduction}

Femtosecond laser machining has enabled the manufacture of a wide variety of integrated, threedimensional, multi-functional devices all on a single substrate [1-12]. While the benefits of this technology are many, there are still significant technological obstacles that must be overcome to reliably and predictably produce these devices. One of these hurdles is that of anisotropy in the writing process.

This anisotropy results from various sources: inhomogeneity in the laser beam [13], pulse front tilt [14], material anisotropy [15], and the formation of selforganized nanostructure [16,17]. Often these effects are manifested as a residual stress. For example, in silica glass $[18,19]$ stress arises form re-arrangement of the material lattice, which is accompanied by a volume change [20-23].

Often, this stress is an un-desirable result, ultimately leading to detrimental effects such as cracking and early device failure in waveguide devices [24,25], as well as increased birefringence and losses [22,26-31]. On the other hand, stress has also been shown to locally influence the etching rate of silica [32,33], potentially resulting in faster processing times. From these examples, it is clear that the process of laser fabrication would greatly benefit from more information about the stress state in a material.

In this work, we focus on decoupling the various contributions to laser-induced anisotropy through the use of highly symmetric laser-written patterns [34]. Here we purposefully introduce anisotropic stress and, through manipulation of the writing parameters, reveal the nature and origin of the anisotropy by measuring the resulting stress-induced birefringence.

\section{Methodology}

We have chosen symmetric laser-written features as the basis for our test structure. Symmetry of the test structure plays an important role, as this aids in revealing directionality effects in the writing process. In practice, these structures are composed of a series of rings, stacked to form a tube. An illustrative example outlining the fabrication process is given in Figure 1.

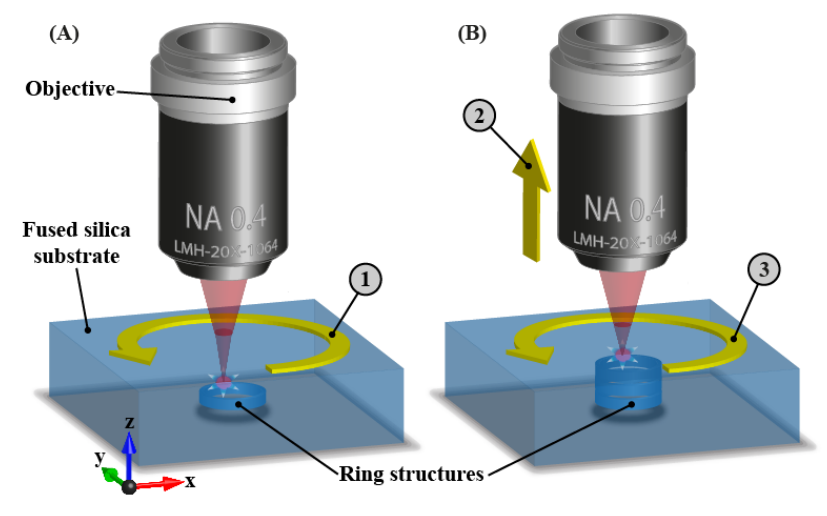

Figure 1. Illustrative example of the process for fabricating test structures used in the investigation of stress anisotropy $(a, b)$ and the result-ing retardance map (c) [34].

Pulses from an ultrafast laser system (Amplitude Systèmes, $1030 \mathrm{~nm}, 275 \mathrm{fs}, 400 \mathrm{kHz}$ ) are focused into a bulk silica substrate using a 20x / 0.4NA objective. The substrate is then moved along a circular path using a set of high-bandwidth flexure stages, forming a ring. To form a tube, successive rings are 'stacked' by translating the focus and repeating the writing of an individual ring. Rings are spaced so that some overlap between

\footnotetext{
${ }^{\mathrm{a}}$ Corresponding author: ben@mcmillen.eu
} 
successive features is present. We refer to this structure as a 'stressor'.

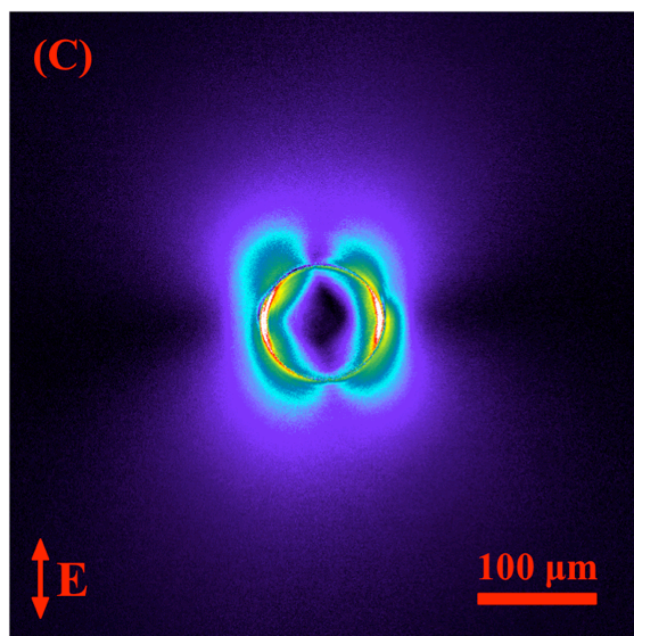

Figure 2. Example retardance map for a tube stressor written with linear polarization [34].

Stress anisotropy is revealed post fabrication by imaging the resulting tubular structures with a standard microscope (Olympus BX-51) equipped with a commercial optical retardance measurement system (CRi-Instruments). False color images are obtained, giving a direct measurement of the induced stress in the sample through photelasticity [35]. Note that, while these measurements give information about the magnitude and orientation of the stress, we primarily focus on the intensity portion of the measurements.

An example retardance map for a tubular feature is illustrated in Figure 2 for a stressor with a diameter of $100-\mu \mathrm{m}$ written in fused silica. The anisotropic pattern shown here is characteristic of the formation of nanogratings within the laser modified zone.

\section{Experimental Results}

\subsection{Fused Silica}

Fused silica presents an interesting material for study, particularly due to its use in the fabrication of a wide variety of devices [2,4,6,32,36,37]. When fabricating these devices, laser-induced anisotropy can have adverse effects on production time, defects, and overall device reliability. The cause of these effects is often the presence of self-organized nanostructure (i.e. "nanogratings"), which are known to form within the laser-modified zone [16].

Using tubular stressors, we can now correlate the formation of these nanogratings with the occurrence of laser-induced stress anisotropy. Given an amorphous material, simply rotating the writing polarization while fabricating successive stressors should reveal a change in the orientation of the induced stress anisotropy.

This hypothesis was tested by writing a series of tubes in a $1-\mathrm{mm}$ thick silica substrate, rotating the polarization between each successive feature. The sample was then imaged and followed by a brief ( $\sim 5$ minute) etch in $2.5 \%$ hydrofluoric acid to reveal any nanostructure that may have formed. The results of this experiment, along with included scanning electron microscope (SEM) images of the etched tubes, are shown in Figure 3. From these images, it is readily apparent that the stress patterns strongly correlate with the presence of nanogratings, particularly when considering the change in orientation of the anisotropic retardance field with respect to the laser polarization.
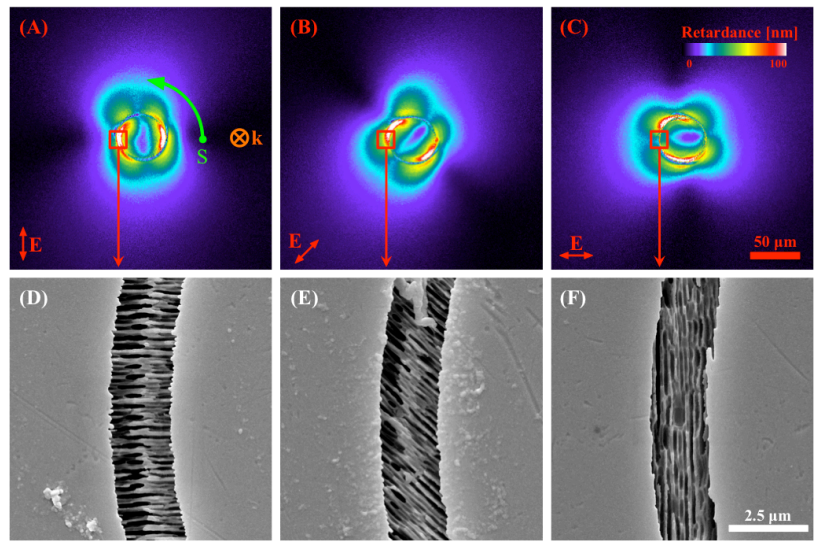

Figure 3. Examples of the resulting anisotropic retardance fields obtained when writing tubes with rotated linear polarization. Corresponding SEM images are provided for correlation with the orientation of the stress field [34].

While tube structures are useful for quick identification of the presence of nanogratings, other directionally dependent anisotropies [14] may not so readily apparent. This is due to several factors. Tube structures themselves are axially symmetric, meaning that any effects arising from directionality will have little effect on the resulting retardance pattern. Furthermore, the induced retardance as a result of the presence of nanogratings is quite large, with stresses between the lamella of the gratings approaching $2 \mathrm{GPa}$ [23]. In this case, it is unlikely that any subtle effects from directionality will be visible.

Instead, we propose the use of so-called 'exotic' polarization states, namely radial and azimuthal polarization. Here the idea is to produce nanogratings whose orientation is directionally independent, and hence any effects such as pulse-front tilt will be more easily identified. Following the methods used in testing the linear case, a series of tubes was again written using three polarization states: circular (for comparison), radial, and azimuthal. These states were generated using a passive polarization converter [36]. The resulting retardance maps and corresponding SEM images are given in Figure 4.

We first note that circular polarization (d) does not yield a truly isotropic retardance map, which is somewhat counterintuitive. Closer examination of the corresponding nanogratings reveals structures that closely resemble the linear polarization case presented in Figure 3. This may be due to several factors, including imperfections in the polarization state of the circularly polarized beam (may have been elliptically polarized), and possibly Fresnel reflections at the air-glass interface. 
This observation is in stark contrast to the retardance maps for radial and azimuthal polarization, which appear nearly perfectly isotropic. Examination of the nanostructure in (b) and (c) reveals gratings that closely mimic the polarization of the writing beam, suggesting that we have in fact successfully generated a truly isotropic pattern.
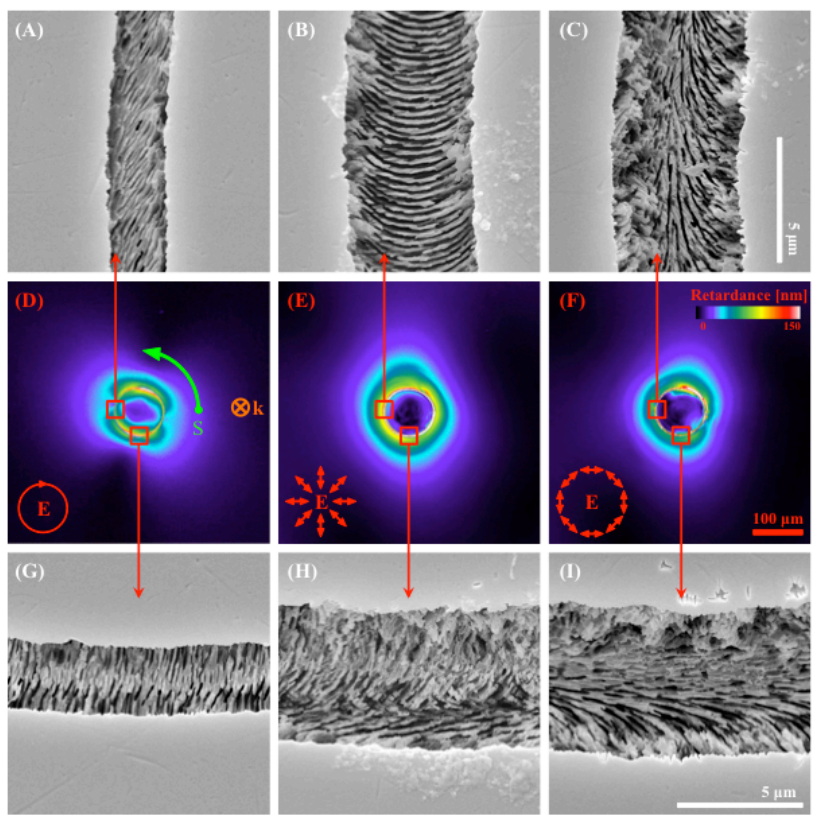

Figure 4. Isotropic stress states obtained by writing tubes with radial and azimuthal polarization (e-f). The feature in (d) was written using circular polarization and serves as a reference. Corresponding SEM images are also given for comparison [34].

However, we note several anomalies in the retardance map at the upper and lower regions of each structure. SEM images reveal that the grating structure is discontinuous and appears as a mixing between two orientations. The irregular structure in these regions causes uneven loading, resulting in slight distortions to the retardance maps shown in (e) and (f).

It should be noted that, when comparing the left (Fig. $4 \mathrm{~b}$ and c) to the right (not shown) side of each structure, the orientation of the nanostructure is the same for both sides (i.e. it does not follow the direction of writing, as indicated by the green arrow). Though we do not yet have a solid explanation for this observation, we theorize that these findings my reveal anisotropy in the writing beam itself.

The results here have primarily focused on the intensity portion of the retardance; however one should keep in mind that additional information has been left out of this measurement. This is illustrated in Figure 5, where the retardance map of a tube written with radial polarization has been overlaid with the corresponding slow-axis orientation. While the retardance field map appears isotropic, the slow axis orientation is not symmetric. A similar image can be obtained for azimuthal polarization.

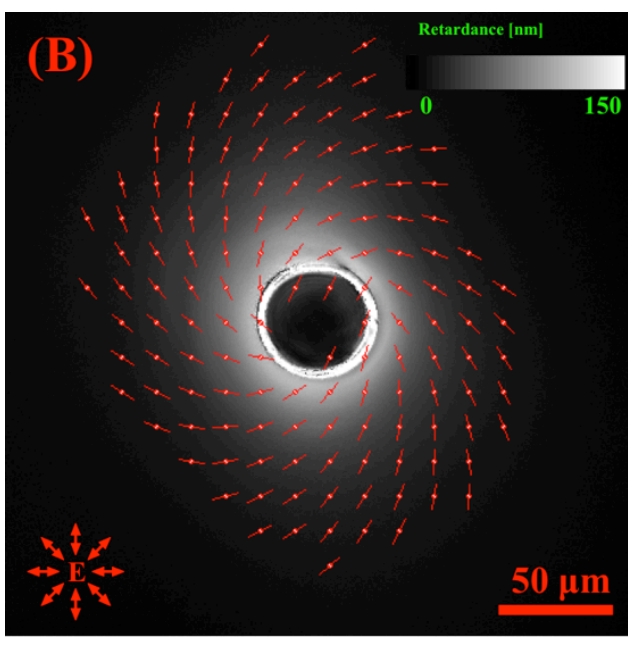

Figure 5. Azimuth (slow-axis orientation) overlay for a tube structure written with radial polarization. Despite the appearance of being isotropic, the retardance map still contains anisotropies that may not be readily visible using this technique [34].

This irregularity gives further information about the nature of the stress generated by the nanograting structure. Referring back to the SEM images shown in Figure 4 and considering that the maximum component of stress is generated perpendicular to the grating lamella, we find that the gratings have an irregular shape, resulting form the use of radial polarization. Since the gratings are organized in a semi-continuous fashion around the tube, we can conclude that the generated stress will be oriented tangentially to the radius of the tube structure. The largest component of stress therefore coincides with the orientation of the slow axis, as shown in the overlay in Figure 5.

\subsection{Crystalline Quartz (c-SiO $)_{2}$}

In the case of silica, tube stressors effectively reveal the presence of self-organized nanostructure. Due to the amorphous structure of this material, no additional information is added to the resulting retardance map. When applied to crystalline quartz however, the result is quite different.

Tubes were again written through the entire thickness of a $125-\mu \mathrm{m}$ z-cut quartz substrate using similar parameters as those for silica, but with the diameter reduced to $25-\mu \mathrm{m}$ to aid in visualization of the retardance patterns. Successive tubes were written with three linear polarization states $(0,45,90$ degrees $)$ as well as radial polarization. The results are given in Figure 6.

We first note that the retardance maps mimic the crystal structure, which is most evident in Figure 6 (d), where radial polarization creates a stress field nearly free of distortion. The corresponding maps for all three linear cases also mimic the structure of the substrate, though with slight distortions corresponding to the formation of nanogratings as shown in (e-g). These distortions, though not readily apparent, also coincide with the orientation of the writing polarization. 

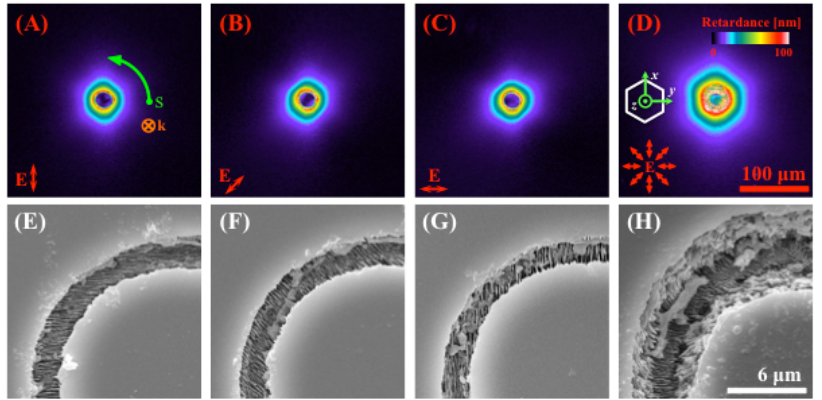

Figure 6. Tubes written in crystalline quartz. The resulting stress field not only mimics the underlying structure but also reveals the presence of nanostructure in the focal volume through distortions in the measured field [34].

Tube stressors can also be used for quantitative analysis, as shown in Figure 7, where contour data has been extracted from the retardance maps shown in Figure 6. For further analysis, angular data has been extracted and plotted in the graphs shown in (e-h), with the location of each angle given in (i). The structure of quartz is given in (j) for reference.

In these plots (a-d), the distortion between each linear case (as compared to the radial polarization case) is much more prominent. Each distortion correlates well with the direction of the laser polarization, particularly when compared with the corresponding angular data in (e-h). In each linear case (a-c), the angular location with the most distortion corresponds to the direction of the maximum anisotropic stress component.
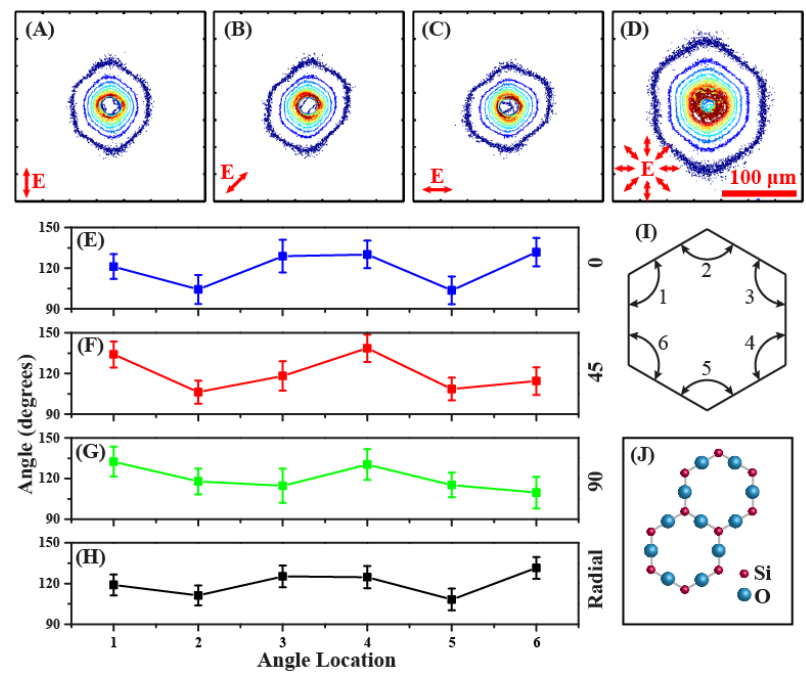

Figure 7. Contour maps (a-d) and corresponding angular data (e-h) for the tube structures presented in Figure 6. In these plots, the distortions are much more noticeable [34].

As with previous tests in silica, radial polarization (d) is used as a reference, showing only slight distortions to the overall pattern. The angular data displayed in (h) shows angles that are approximately equal $(120+/-10$ degrees). Here, the small differences are attributed to the previously observed nanograting anomalies in the upper and lower regions of the ring (see Figure 4(h), for example), as well as differences in Young's modulus for the $x$ - and $y$-directions in the crystal.

\subsection{Nd:YAG}

In the case of quartz, perhaps the most notable outcome of the previous experiment was that the retardance patterns closely mimic the structure of the underlying crystal system. With this in mind, the same tube-based analysis technique was applied to other crystal systems. In this section, we focus on Nd:YAG, which has a cubic structure.

Tubes were written at a depth of $200 \mu \mathrm{m}$, using an exploratory pulse energy sweep of $100-300 \mathrm{~nJ}$ per pulse. As the basis of this experiment was to reveal the crystal structure, only linear polarizations of 0 and 90 degrees were used. The results of this experiment are shown in Figure 8.

Starting with the retardance map shown in (b) for the case of 'horizontal' writing polarization, we again find that the stress in the substrate very closely mimics the crystal structure. Perhaps the most striking feature is the apparent lack of retardance for the 'vertical' polarization case shown in (a) despite identical writing conditions to those of (b). As a comparison, the same features were imaged under crossed polarizers, as shown in Figure 8(c) and (d), to rule out the possibilities of any measurement anomalies from using the CRi retardance measurement system.
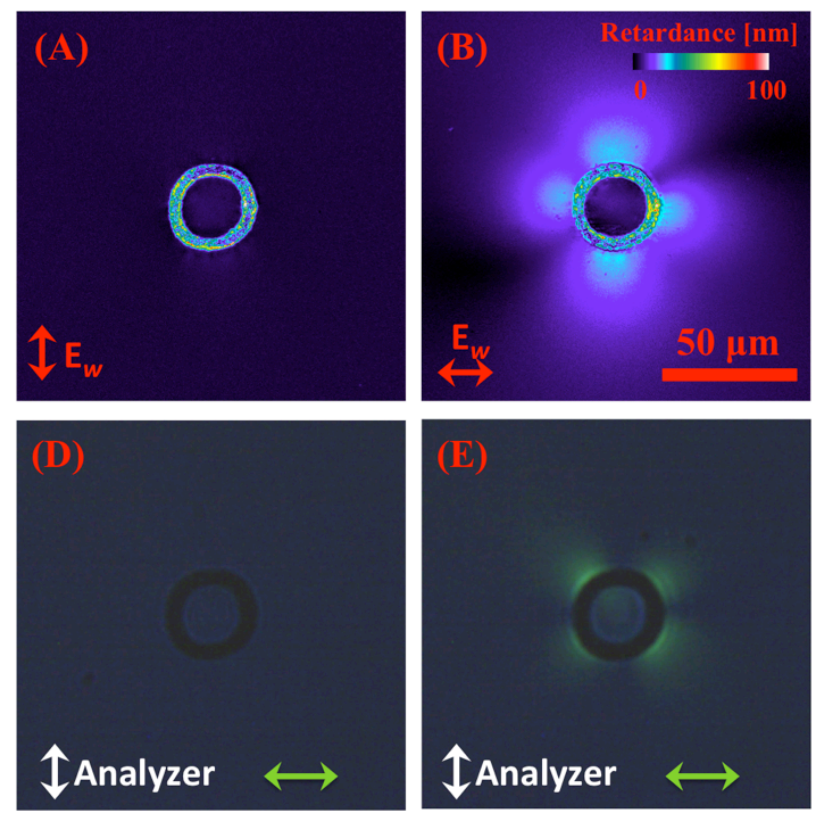

Figure 8. Tubes written in Nd:YAG, showing the difference between orthogonal writing polarizations $(\mathrm{a}-\mathrm{b})$. In (b) the cubic structure of the material is clearly visible, while in (a) no stress is present despite similar writing conditions.

To gain more insight into this observation, SEM images were also taken of the same damage sites, as shown in Figure 9. Between the two features (written with 'vertical' and 'horizontal' polarization respectively), no apparent differences can be found in the morphology of the structure. It should be noted here that the substrate was not etched prior to imaging. In the course of our work involving laser modification of dielectrics, we have found that in some cases, nanogratings are visible on the 
surface after machining, requiring no post-development step (i.e. etching).
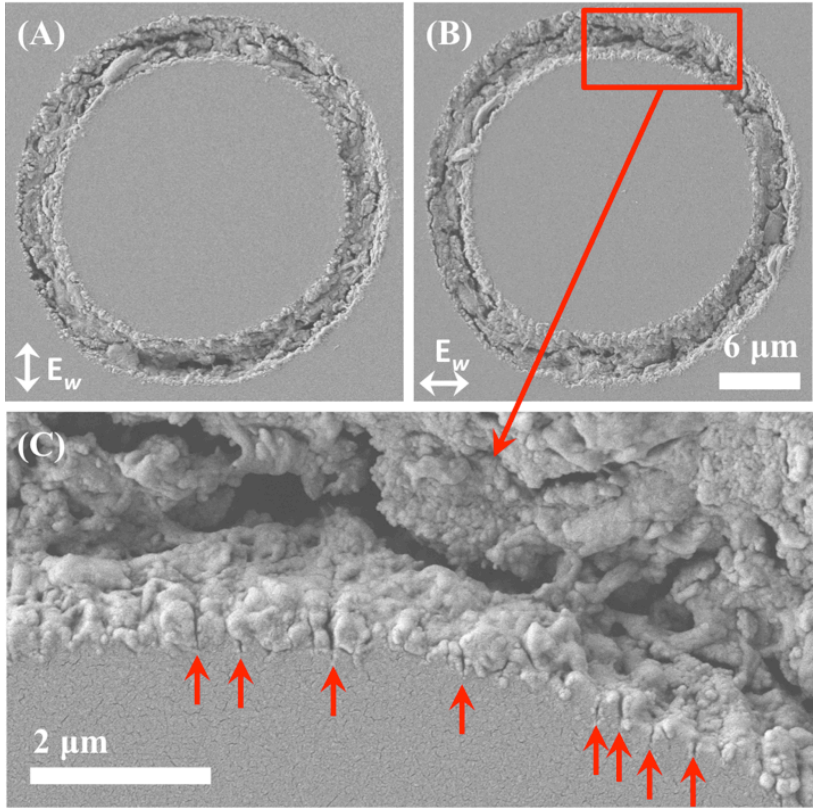

Figure 9. SEM images of surface structure of tubes written in Nd:YAG. No nanostructure was found for either writing polarization (a-b), though the magnified view in (c) suggests that nanogratings may have formed during the fabrication process but were subsequently destroyed following further laser exposure.

Despite the apparent lack of differences, some clues are evident in the magnified region shown in Figure 9(c). Here we find vertically oriented cracks, which appear to have a semi-regular arrangement. Additionally, the orientation is perpendicular to the polarization of the writing beam. Similar features were found for the 'vertical' polarization case shown in (a), but no obvious nanostructure formation was found, even for lower pulse energies. The investigation of these anomalies is currently ongoing.

\section{Modelling}

The characteristic anisotropic retardance shown in Figure 3 may be explained with the help of a simple model, which is displayed in Figure 10(a). Here we have graphically represented one quarter of a tube structure, showing the orientation of nanogratings with respect to a 'vertical' writing polarization, along with the deformation developed with respect to the x-axis (shown as the red curve). The inset in (a) shows the stress developed by a single grating lamella.

This model is based on work by Champion et al, in which nanogratings were shown to be responsible for a deformation of approximately $\sim 0.03 \%$ [23]. It was also shown that the maximum component of stress is generated in the direction perpendicular to the grating lamella (parallel to the writing polarization). Taking these factors into account, we can now treat the laser-modified material within each ring using a 'bulk' approach. By calculating the chord length between the inner and outer radius (either parallel or perpendicular to the grating lamella), we now arrive at the deformation profile given in (a). Note that the peak in this curve represents a discontinuity, which is found when the angularly dependent $x$-position coincides with the inner radius, yielding a maximum value of the chord length, and hence a peak in the deformation profile.
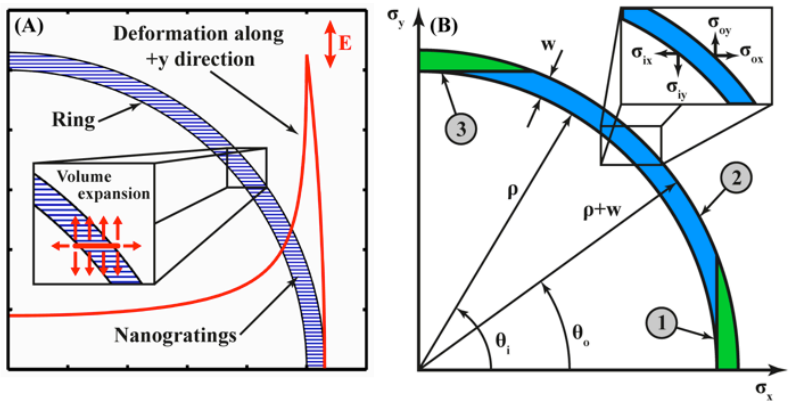

Figure 10. Nanograting stress model for tubes in silica [34].

The plot in Figure 10(b) takes the simplified model in (a) one step further, formalizing this derivation for stresses acting on the inner and outer radius $\left(\sigma_{i}\right.$ and $\sigma_{o}$ respectively) as a function of angle along both the $x$ - and $y$-directions. The three regions (labeled 1, 2, and 3) represent regions of discontinuity where the boundary conditions change with respect to the inner and outer radii. Due to the difference in stress magnitude generated either perpendicular or parallel to the grating lamella, a ratio factor was also included in the model. For more information, the full derivation is published elsewhere [34].
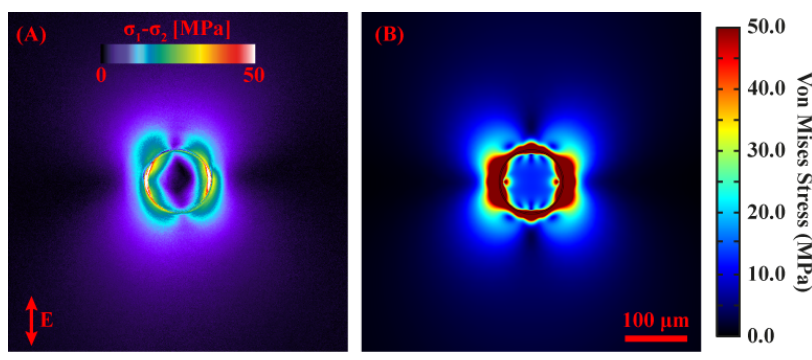

Figure 11. Comparison between the retardance map of a tube structure in silica (which has been scaled to display difference principal stress) and the simulation result using the model shown in Figure 10 [34].

This model was verified using COMSOL for a tube with an outer diameter of $100 \mu \mathrm{m}$ and a laser-modified zone width of $2 \mu \mathrm{m}$, and treated as a plane-strain problem due to the high aspect ratio of a typical tube structure. Based on findings by Champion et al [23], the peak stress was scaled to a maximum value of $2 \mathrm{GPa}$ with the ratio between $x$ - and $y$-components set to 2.5 . The result is shown in Figure 11(b).

Figure 11 also includes a representative retardance map (a) for a tube with a diameter of $100 \mu \mathrm{m}$, which has been scaled to show difference principal stress according to the following equation [35]: 


$$
\sigma_{1}-\sigma_{2}=\frac{R}{T\left(C_{1}-C_{2}\right)}
$$

where the difference principal stress is given by the quantity $\sigma_{l}-\sigma_{2}, R$ is the measured retardance, and $T$ the thickness of the material. The constant $C_{1}-C_{2}=3.55 \times 10^{-12}$ $\mathrm{Pa}$ and is derived from the piezo-optical coefficients of fused silica through the following relationship [35]:

$$
C=\left(\frac{n^{3}}{2}\right)\left(\pi_{11}-\pi_{12}\right) .
$$

Comparison between the real measurement (a) and the simulated model (b) is made using the largest part of the 'lobes' (colored light blue / green in (a)). Here we find that the maximum stress approaches $20 \mathrm{GPa}$, which is in good agreement with the model shown in (b).

The model is overall in good agreement with the original tube structure, however some subtle differences are readily noticeable. In particular, the stress concentrations in the 'lobes' of the model are broader and longer than those of the original tube structure. This difference may be accounted for by considering that the laser-modified zone has been heavily transformed, and as such, cannot be treated as bulk material. In the model, the ring structure was used merely to define the stress profile around the laser-modified zone. Since we do not yet know the mechanical properties of this region, they were assumed to be the same as the bulk substrate.

\section{Future Outlook and Conclusions}

Until now we have discussed laser-induced stress anisotropy as it relates the outcome of laser fabrication of devices. This effect may be considered in another light, especially given the toolset presented here. Tube-based stressors, along with a predictive model, represent a means by which stress may be generated as a useful outcome, leading to new optical devices based on stressinduced birefringence.

Figure 12 shows several examples of the use of tube structures to generate complex retardance patterns. The patterns shown in (a-c) are representative of basic geometric layouts with the corresponding tube position and writing polarization shown below. These layouts are for two-dimensional patterns, however three-dimensional configurations are also possible, as shown in (d). Here tubes are written in groups of 8 rings while successively rotating the polarization 15 degrees between each group, resulting in a retardance pattern with a vortex-like shape.

While these structures are rather simplistic, they serve as an example of the possibilities of using laserinduced stress to create new optical devices, particularly in the area of complex polarization generation [36]. If properly designed, these structures allow for an optical device in which the propagating beam encounters only pristine bulk material, which has been modified only through the secondary action of stress generated with the tube structures. This not only reduces losses from absorption and scattering, but also increases the powerhandling capability of such a device.
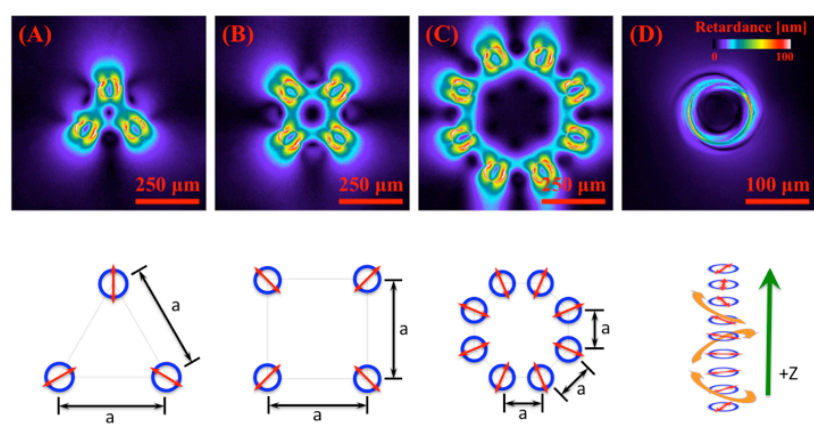

Figure 12. Examples of stress induced retardance patterns using tube structures.

The role of stress in laser-modified materials plays a fundamental role in the process of laser machining. Through the techniques presented here, we aim to provide another tool by which the researcher may further process and analyze any given dielectric material suitable for laser processing.

Here we have presented a methodology to quickly identify and quantify the presence of anisotropies in the laser writing process. Through the use of retardance imaging, we rely on the generation of stress in the substrate through the formation of nanogratings (in amorphous materials) or disruption of the material lattice (in crystals) as a means to gauge the effects of laser machining on a given substrate.

Additionally, we have presented a model with may be used as a predictive tool, specifically in the case of nanograting formation in silica glass. Though we have only presented a model for amorphous materials, this methodology may be further extended to that of crystalline substrates.

\section{Acknowledgements}

The authors acknowledge the financial support from the European Research Council under the Galatea Project (ERC-2012-StG-307442), http://www.erc-galatea.eu. The authors also acknowledge Prof. Peter Kazansky from the University of Southampton for the use of his polarization converter.

\section{References}

1. Y. Cheng, K. Sugioka, K. Midorikawa, M. Masuda, K. Toyoda, M. Kawachi, and K. Shihoyama, Opt. Lett. 28, 55-57 (2003).

2. K. Sugioka, Y. Hanada, and K. Midorikawa, Laser \& Photon. Rev. 4, 386-400 (2010).

3. J. W. Chan, T. R. Huser, S. H. Risbud, J. S. Hayden, and D. M. Krol, Appl. Phys. Lett. 82, 2371-2373 (2003).

4. K. Minoshima, A. Kowalevicz, E. Ippen, and J. Fujimoto, Opt. Express 10, 645 (2002).

5. G. A. Torchia, C. Mendez, I. Arias, L. Roso, A. Rodenas, and D. Jaque, Appl. Phys. B-Lasers O. 83, 559-563 (2006). 
6. Y. Sikorski, A. A. Said, P. Bado, R. Maynard, C. Florea, and K. A. Winick, Electron. Lett. 36, 226227 (2000).

7. G. A. Torchia, P. F. Meilán, A. Ródenas, D. Jaque, C. Mendez, and L. Roso, Opt. Express 15, 1326613271 (2007).

8. P. Nandi, G. Jose, C. Jayakrishnan, and S. Debbarma, Opt. Express 14, 12145-12150 (2006).

9. Y. Tokuda, M. Saito, M. Takahashi, and K. Yamada, J. Non-Cryst. Solids 326-327, 472-475 (2003).

10. S. Juodkazis and H. Misawa, Appl. Phys. A-Mater. 93, 857-861 (2008).

11. B. McMillen, K. Chen, Honglin An, Simon Fleming, V. Hartwell, and D. Snoke, Appl. Phys. Lett. 93, 111106-111103 (2008).

12. S. Hasegawa and Y. Hayasaki, Int. J. Optomech. 8, 73-88 (2014).

13. P. S. Salter and M. J. Booth, Appl. Phys. Lett. 101, 141109 (2012).

14. P. Kazansky, W. Yang, E. Bricchi, and J. Bovatsek, Appl. Phys. Lett. 90, 151120 (2007).

15. W. Yang, P. G. Kazansky, and Y. P. Svirko, Nature Photon. 2, 99-104 (2008).

16. Y. Shimotsuma, P. Kazansky, and J. Qiu, Phys. Rev. Lett. 91, 247405 (2003).

17. V. R. Bhardwaj, E. Simova, P. P. Rajeev, C. Hnatovsky, R. S. Taylor, D. M. Rayner, and P. B. Corkum, Phys. Rev. Lett. 96, 057404 (2006).

18. B. Poumellec, L. Sudrie, M. Franco, B. Prade, and A. Mysyrowicz, Opt. Express 11, 1070-1079 (2003).

19. A. Champion, M. Beresna, P. Kazansky, and Y. Bellouard, Opt. Express 21, 24942-24951 (2013).

20. E. N. Glezer and E. Mazur, Appl. Phys. Lett. 71, 882-884 (1997)

21. Y. Bellouard, A. Said, M. Dugan, and P. Bado, Opt. Express 12, 2120 (2004).
22. Y. Bellouard, T. Colomb, C. Depeursinge, and M. Dugan, Opt. Express 14, 8360 (2006).

23. A. Champion and Y. Bellouard, Opt. Mater. Express 2, 789-798 (2012).

24. A. Champion, Y. Bellouard, G. Mindaugas, M. Beresna, and P. G. Kazansky, Proc. SPIE 7925, (2011).

25. A. Rodenas, A. Benayas, J. R. Macdonald, J. Zhang, D. Y. Tang, D. Jaque, and A. K. Kar, Opt. Lett. 36, 3395-3397 (2011).

26. J. Bai, G. Cheng, X. Long, Y. Wang, W. Zhao, G. Chen, R. Stoian, and R. Hui, Opt. Express 20, 15035-15044 (2012).

27. V. Bhardwaj, P. Corkum, D. Rayner, C. Hnatovsky, E. Simova, and R. Taylor, Opt. Lett. 29, 1312-1314 (2004).

28. D. Grobnic, S. J. Mihailov, and C. W. Smelser, J. Lightwave Technol., 25, 1996-2001 (2007).

29. P. Yang, G. R. Burns, J. Guo, T. S. Luk, and G. A. Vawter, J. Appl. Phys. 95, 5280-5283 (2004).

30. L. Sudrie, M. Franco, and B. Prade, Opt. Commun. 191, 333-339 (2001).

31. G. Zhou, A. Jesacher, M. Booth, T. Wilson, and A. Rodenas, Opt. Express 17, 17970-17975 (2009).

32. F. Madani-Grasset and Y. Bellouard, Opt. Express 18, 21826-21840 (2010).

33. M. Tomozawa and T. Takamori, J. Am. Ceram. Soc. 62, 370-373 (1979).

34. B. McMillen and Y. Bellouard, Opt. Express 23, 86 (2015).

35. J. F. Nye, Physical Properties of Crystals (Oxford University Press, 1985)

36. M. Beresna, M. Gecevičius, P. G. Kazansky, and T. Gertus, Appl. Phys. Lett. 98, 201101 (2011).

37. B. Lenssen and Y. Bellouard, Appl. Phys. Lett. 101, 103503-4 (2012). 